Algebraic 85 Geometric Topology

Volume 1 (2001) 699-708

Published: 22 November 2001

\title{
The mapping class group of a genus two surface is linear
}

\author{
Stephen J. Bigelow \\ RYAN D. BUDNEY
}

\begin{abstract}
In this paper we construct a faithful representation of the mapping class group of the genus two surface into a group of matrices over the complex numbers. Our starting point is the Lawrence-Krammer representation of the braid group $B_{n}$, which was shown to be faithful by Bigelow and Krammer. We obtain a faithful representation of the mapping class group of the $n$-punctured sphere by using the close relationship between this group and $B_{n-1}$. We then extend this to a faithful representation of the mapping class group of the genus two surface, using Birman and Hilden's result that this group is a $\mathbf{Z}_{2}$ central extension of the mapping class group of the 6 -punctured sphere. The resulting representation has dimension sixty-four and will be described explicitly. In closing we will remark on subgroups of mapping class groups which can be shown to be linear using similar techniques.
\end{abstract}

AMS Classification 20F36; 57M07, 20C15

Keywords Mapping class group, braid group, linear, representation

\section{Introduction}

Let Diff $M$ denote the topological group of orientation preserving diffeomorphisms of an oriented manifold $M$ which act as the identity on $\partial M$. The mapping class group of $M$ is the group $\pi_{0} \operatorname{Diff} M$. A representation of a group is a homomorphism from the group into a multiplicative group of matrices over some commutative ring. A representation is called faithful if it is one-to-one. A group is called linear if it admits a faithful representation.

The aim of this paper is to construct a faithful representation of the mapping class group of the genus two surface. In the process we construct faithful representations of mapping class groups of punctured spheres, hyperelliptic mapping class groups and, more generally, normalizers of certain covering transformation groups of surfaces. 
We take as our starting point the Lawrence-Krammer representation of the braid group $B_{n}$. Bigelow [Big] and Krammer [Kra2] have shown this to be faithful. In Section 2, we show how to alter the Lawrence-Krammer representation to obtain a faithful representation of the mapping class group of an $n$-times punctured sphere.

The genus two surface is a branched covering space of the sphere with six branch points. Birman and Hilden $[\mathrm{BH}]$ have used this fact to establish a close relationship between the mapping class group of the genus two surface and the mapping class group of the six-times punctured sphere. In Section 3, we use this relationship to obtain a faithful representation of the mapping class group of the genus two surface.

Simultaneous with this result, Nathan Dunfield and also Mustafa Korkmaz [Kor] have individually produced faithful representations of the mapping class group of the genus two surface. All of these constructions use the relationship to the mapping class group of the six-times punctured sphere. However we have taken a bit of extra care to keep the dimension reasonably low. Our faithful representations of the mapping class groups of the $n$-times punctured sphere and the genus two surface have dimensions $n\left(\begin{array}{c}n-1 \\ 2\end{array}\right)$ and 64 respectively, whereas the representations in [Kor] have dimensions $n\left(\begin{array}{c}n-1 \\ 2\end{array}\right)$ and $2^{10} 3^{5} 5^{3}$ respectively.

The low rank of our representation makes it suitable for computer use, and we explicitly compute the matrices for our representations in Section 4. In Section 5 we show how to generalize our construction to obtain faithful representations normalizers of a class of finite subgroups of mapping class groups. The simplest such generalization gives a faithful representation of the hyperelliptic group of the genus $g$ surface. Korkmaz [Kor] also constructed a faithful representation of the hyperelliptic group, but once again ours has a smaller dimension, namely $(2 g+2)\left(\begin{array}{c}2 g+1 \\ 2\end{array}\right)+2 g$ as opposed to $(2 g+2)\left(\begin{array}{c}2 g+1 \\ 2\end{array}\right){ }^{2} 3^{g^{2}} \prod_{i=1}^{g}\left(3^{2 i}-1\right)$.

Throughout this paper, $D$ will denote a disk, $\Sigma_{2}$ will denote a closed oriented surface of genus two, and $S^{2}$ will denote a sphere. If $M$ is an oriented manifold and $n$ is a positive integer then let $\operatorname{Diff}(M, n)$ denote $\operatorname{Diff}\left(M,\left\{p_{1}, \ldots, p_{n}\right\}\right)$, where $p_{1}, \ldots, p_{n}$ are distinct points in the interior of $M$. This is the group of diffeomorphisms of $M$ that restrict to permutations of the set $\left\{p_{1}, \ldots, p_{n}\right\}$. 


\section{The $n$-punctured Sphere}

The aim of this section is to prove the following.

Theorem 2.1 There exists a faithful representation of the mapping class group of the $n$-times punctured sphere.

The braid group $B_{n}$ is the group $\pi_{0} \operatorname{Diff}(D, n)$. Provided $n \geq 3$, the center of $B_{n}$ is isomorphic to $\mathbf{Z}$ and is generated by the full twist braid $\Delta^{2}$. This is a Dehn twist about a curve which is parallel to $\partial D$.

Let $p_{1}, \ldots, p_{n}$ be distinct points in $S^{2}$.

Lemma 2.2 Provided $n \geq 4$, there is a short exact sequence

$$
0 \rightarrow \mathbf{Z} \rightarrow B_{n-1} \rightarrow \operatorname{Stab}\left(p_{n}\right) \rightarrow 0,
$$

where the image of $\mathbf{Z}$ in $B_{n-1}$ is the center of $B_{n-1}$, and $\operatorname{Stab}\left(p_{n}\right)$ is the subgroup of $\pi_{0} \operatorname{Diff}\left(S^{2}, n\right)$ consisting of diffeomorphisms that fix the point $p_{n}$.

Proof Let $D^{+}$and $D^{-}$be the northern and southern hemispheres of $S^{2}$, that is, two disks in $S^{2}$ such that $D^{+} \cap D^{-}=\partial D^{+}=\partial D^{-}$. Assume that $p_{1}, \ldots, p_{n-1} \in D^{+}$and $p_{n} \in D^{-}$. Then $B_{n-1}$ is $\pi_{0} \operatorname{Diff}\left(D^{+}, n-1\right)$. We can extend any $f \in \operatorname{Diff}\left(D^{+}, n-1\right)$ to a diffeomorphism of the whole sphere by setting it to be the identity on $D^{-}$. Let $\phi: B_{n-1} \rightarrow \pi_{0} \operatorname{Diff}\left(S^{2}, n\right)$ be the homomorphism defined in this way. This will be the rightmost map in our short exact sequence.

First we show that the image of $\phi$ is $\operatorname{Stab}\left(p_{n}\right)$. Let $g$ be an element of $\operatorname{Diff}\left(S^{2}, n\right)$ which fixes the puncture $p_{n}$. Note that $g_{\mid\left(D^{-}\right)}$is a closed tubular neighborhood of $p_{n}$ in $S^{2}-\left\{p_{1}, \cdots, p_{n-1}\right\}$. By the uniqueness of tubular neighborhoods theorem, $g_{\mid\left(D^{-}\right)}$is isotopic to the identity relative to $\left\{p_{n}\right\}$. This isotopy can be extended to an ambient isotopy of the $n$-times punctured sphere. We can therefore assume, without loss of generality, that $g$ acts as the identity on $\partial D^{-}$. Thus $g=\phi\left(g_{\mid\left(D^{-}\right)}\right)$.

Now we show that the kernel of $\phi$ is generated by $\Delta^{2}$. Let $f \in \operatorname{Diff}\left(D^{+}, n-1\right)$ represent an element of the kernel of $\phi$. Let $g=\phi(f)$ be its extension to $S^{2}$ which is the identity on $D^{-}$. Then there is an isotopy $g_{t} \in \operatorname{Diff}\left(S^{2}, n\right)$ such that $g_{0}=g$ and $g_{1}$ is the identity map. Now $g_{t}$ restricted to $D^{-}$defines an element of the fundamental group of the space of all tubular neighborhoods of $p_{n}$. The proof of the uniqueness of tubular neighborhoods theorem [Hir] naturally 
extends to a proof that there is a homotopy equivalence between the space of tubular neighbourhoods of a point and $\operatorname{GL}\left(T_{p_{n}}\right)$. Thus the fundamental group of the space of tubular neighbourhoods of a fixed point in $S^{2}$ is $\mathbf{Z}$, generated by a rigid rotation through an angle of $2 \pi$. Consequently our family of diffeomorphisms $g_{t}$ can be isotoped relative to endpoints so that its restriction to $D^{-}$is a rigid rotations by some multiple of $2 \pi$. Therefore $f$ is isotopic to some power of $\Delta^{2}$.

Let

$$
\mathcal{L}_{n}: B_{n} \rightarrow \mathrm{GL}\left(\left(\begin{array}{l}
n \\
2
\end{array}\right), \mathbf{Z}\left[q^{ \pm 1}, t^{ \pm 1}\right]\right)
$$

denote the Lawrence-Krammer representation, which was shown to be faithful in [Big] and [Kra2]. By assigning algebraically independent complex values to $q$ and $t$, we consider the image as lying in $\operatorname{GL}\left(\left(\begin{array}{l}n \\ 2\end{array}\right), \mathbf{C}\right)$.

Now $\mathcal{L}_{n}\left(\Delta^{2}\right)$ is a scalar matrix $\lambda I$. This can be seen by looking at the representation as an action on the module of forks [Kra1]. (In fact, $\lambda=q^{2 n} t^{2}$.) We will now "rescale" the representation $\mathcal{L}_{n}$ so that $\Delta^{2}$ is mapped to the identity matrix.

The abelianization of $B_{n}$ is $\mathbf{Z}$. Let ab: $B_{n} \rightarrow \mathbf{Z}$ denote the abelianization map. Then $\operatorname{ab}\left(\Delta^{2}\right) \neq 0$, as is easily verified using the standard group presentation for $B_{n}$. (In fact, $\operatorname{ab}\left(\Delta^{2}\right)=n(n-1)$.) Let exp : $\mathbf{Z} \rightarrow \mathbf{C}^{*}$ be a group homomorphism which takes $\operatorname{ab}\left(\Delta^{2}\right)$ to $\lambda^{-1}$. We now define a new representation $\mathcal{L}_{n}^{\prime}$ of $B_{n}$ by

$$
\mathcal{L}_{n}^{\prime}(\beta)=(\exp \circ \mathrm{ab}(\beta)) \mathcal{L}_{n}(\beta) .
$$

We claim that the kernel of $\mathcal{L}_{n}^{\prime}$ is precisely the center of $B_{n}$, provided $n \geq 3$. By design, $\mathcal{L}_{n}^{\prime}\left(\Delta^{2}\right)=I$. Conversely, suppose $\mathcal{L}_{n}^{\prime}(\beta)=I$. Then $\mathcal{L}_{n}(\beta)$ is a scalar matrix, so lies in the center of the matrix group. Since $\mathcal{L}_{n}$ is faithful, it follows that $\beta$ lies in the center of the braid group.

We are now ready to prove Theorem 2.1. If $n \leq 3$ then $\operatorname{Diff}\left(S^{2}, n\right)$ is simply the full symmetric group on the puncture points, so the result is trivial. We therefore assume $n \geq 4$. By Lemma 2.2, $\mathcal{L}_{n-1}^{\prime}$ induces a faithful representation of $\operatorname{Stab}\left(p_{n}\right)$. Since $\operatorname{Stab}\left(p_{n}\right)$ has finite index in $\pi_{0} \operatorname{Diff}\left(S^{2}, n\right), \mathcal{L}_{n-1}^{\prime}$ can be extended to a finite dimensional representation $\mathcal{K}_{n}$ of $\pi_{0} \operatorname{Diff}\left(S^{2}, n\right)$. Extensions of faithful representations are faithful (see for example [Lan]), giving the result.

Note that the faithful representation $\mathcal{K}_{n}$ has dimension $n\left(\begin{array}{c}n-1 \\ 2\end{array}\right)$. 


\section{The genus two surface}

The aim of this section is to prove the following.

Theorem 3.1 There exists a faithful representation of the mapping class group of the genus two surface.

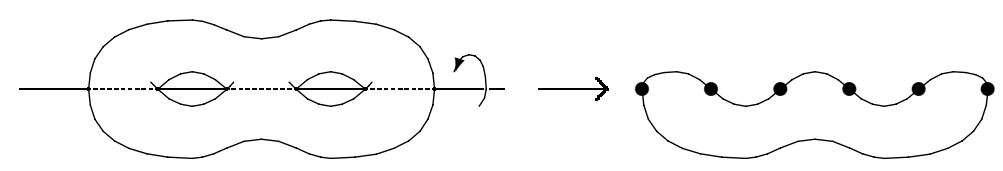

Figure 1: The action of $\mathbf{Z}_{2}$ on $\Sigma_{2}$.

The standard involution of $\Sigma_{2}$ is the rotation through an angle of $\pi$ as shown in Figure 1. This defines an action of $\mathbf{Z}_{2}$ as a group of branched covering transformations with quotient $S^{2}$ and six branch points. Let Diff $\mathbf{Z}_{2} \Sigma_{2}$ denote the group of $\mathbf{Z}_{2}$-equivariant diffeomorphisms of $\Sigma_{2}$, that is, the group of diffeomorphisms which strictly commute with the standard involution. We think of Diff $\mathbf{Z}_{2} \Sigma_{2}$ as a subspace of Diff $\Sigma_{2}$.

Proposition 3.2 The inclusion map Diff $\mathbf{Z}_{2} \Sigma_{2} \rightarrow$ Diff $\Sigma_{2}$ induces an isomorphism on $\pi_{0}$.

Proof That the induced map is epic follows from Lickorish's theorem [Lic] that that the genus two mapping class group is generated by five Dehn twists, all of which happen to be $\mathbf{Z}_{2}$ equivariant. See Figure 2. This is the point where the analogous theorem fails for higher genus surfaces. That the induced map is

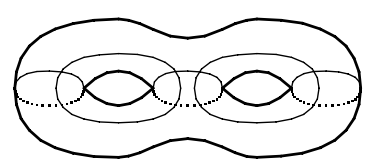

Figure 2: Dehn twists generating the mapping class group of $\Sigma_{2}$.

one-to-one is more difficult. A proof can be found in $[\mathrm{BH}]$.

Proposition 3.3 The quotient map $\operatorname{Diff}^{\mathbf{Z}_{2}} \Sigma_{2} \rightarrow \operatorname{Diff}\left(S^{2}, 6\right)$ induces a short exact sequence

$$
0 \rightarrow \mathbf{Z}_{2} \rightarrow \pi_{0} \operatorname{Diff}^{\mathbf{Z}_{2}} \Sigma_{2} \rightarrow \pi_{0} \operatorname{Diff}\left(S^{2}, 6\right) \rightarrow 0,
$$

where the generator of $\mathbf{Z}_{2}$ is mapped to the standard involution of $\Sigma_{2}$. 
Proof Onto is easy: Each of the five Dehn twists shown in Figure 2 is sent to a half Dehn twist around a curve separating two puncture points from the rest. Two examples are shown in Figure 3. The definition of a half Dehn twist is as

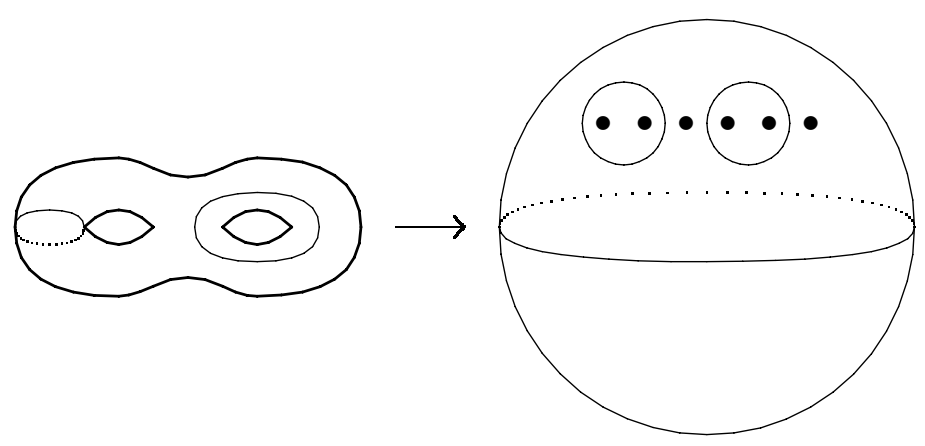

Figure 3: Dehn twists mapped to half Dehn twists.

illustrated in Figure 4. These half Dehn twists are the standard generators of the mapping class group of the 6 -times punctured sphere.

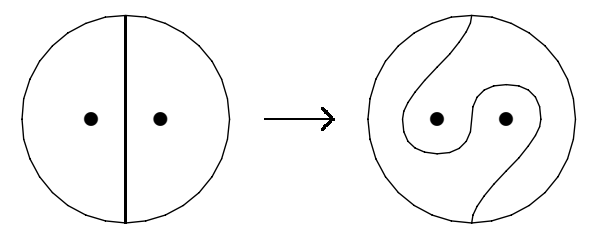

Figure 4: A half Dehn twist

That the kernel is $\mathbf{Z}_{2}$ is an elementary exercise in (branched) covering space theory.

In Section 2 we constructed a faithful representation $\mathcal{K}_{n}$ of $\pi_{0} \operatorname{Diff}\left(S^{2}, n\right)$. By the previous two propositions, $\mathcal{K}_{6}$ is a representation of $\pi_{0}$ Diff $\Sigma_{2}$ whose kernel is equal to $\mathbf{Z}_{2}$, generated by the standard involution.

Let $\mathcal{H}$ be the representation of $\pi_{0} \operatorname{Diff} \Sigma_{2}$ induced by the action of Diff $\Sigma_{2}$ on $H_{1} \Sigma_{2}$. This is called the symplectic representation. Under this representation, the standard involution is sent to $-I$. The direct sum $\mathcal{K}_{6} \oplus \mathcal{H}$ is therefore a faithful representation of $\pi_{0}$ Diff $\Sigma_{2}$. It has dimension $6\left(\begin{array}{l}5 \\ 2\end{array}\right)+4=64$. 


\section{Matrices}

We start off by computing matrices for the representation $\mathcal{L}_{n}^{\prime}$. Explicit matrices for $\mathcal{L}_{n}$ were worked out both in Krammer and Bigelow's work. We use the conventions of $[\mathrm{Big}]$, but we correct a sign error which occurs in that paper. Here, $\sigma_{i}$ are the half Dehn twist generators of the mapping class group of a punctured disk, and $\mathcal{L}_{n}\left(\sigma_{i}\right)$ acts on the vector space $V$ with basis $v_{j, k}$ for $1 \leq j<k \leq n$.

$$
\mathcal{L}_{n}\left(\sigma_{i}\right) v_{j, k}= \begin{cases}v_{j, k} & i \notin\{j-1, j, k-1, k\}, \\ q v_{i, k}+\left(q^{2}-q\right) v_{i, j}+(1-q) v_{j, k} & i=j-1 \\ v_{j+1, k} & i=j \neq k-1, \\ q v_{j, i}+(1-q) v_{j, k}-\left(q^{2}-q\right) t v_{i, k} & i=k-1 \neq j, \\ v_{j, k+1} & i=k, \\ -t q^{2} v_{j, k} & i=j=k-1 .\end{cases}
$$

Using this, we can compute $\exp \circ \mathrm{ab}\left(\sigma_{i}\right)=t^{-1 / d} q^{-n / d}$, with $d=\left(\begin{array}{l}n \\ 2\end{array}\right)$. Consequently, $\mathcal{L}_{n}^{\prime}\left(\sigma_{i}\right)=t^{-1 / d} q^{-n / d} \mathcal{L}_{n}\left(\sigma_{i}\right)$.

The induced representation $\mathcal{K}_{n}$ of $\mathcal{L}_{n-1}^{\prime}$ is now straightforward to compute, and we will give a block-matrix description of it in terms of $\mathcal{L}_{n-1}^{\prime}$.

Reminder: suppose a subgroup $A$ of a group $B$ acts on a vector space $V$. The induced representation of $B$ is the module $\operatorname{Map}^{A}(B, V)$ of $A$-equivariant maps from $B$ to $V$. The action of $B$ on this module is given by $b . f:=f \circ R_{b}$, where $R_{b}: B \rightarrow B$ is right multiplication by $b$. Let $\left\{c_{i}\right\}$ be a set of coset representatives of $A$ in $B$, ie., $B$ is the disjoint union of the cosets $c_{i} A$. Then $\operatorname{Map}^{A}(B, V)=\oplus_{i} c_{i} . V$, where our inclusion $V \longmapsto \operatorname{Map}^{A}(B, V)$ is given by the $A$-equivariant maps from $B$ to $V$ which are zero outside of $A$. The direct sum is in the category of abelian groups. See [Lan, Proposition XVIII.7.2] for details.

As coset representatives for $\operatorname{Stab}\left(p_{n}\right)$ in $\pi_{0} \operatorname{Diff}\left(S^{2}, n\right)$ we will use the maps $c_{1}=I d, c_{2}=\sigma_{n-1}$, and

$$
c_{i}=\left(\sigma_{n-i+1} \sigma_{n-i+2} \ldots \sigma_{n-2}\right) \sigma_{n-1}\left(\sigma_{n-i+1} \sigma_{n-i+2} \ldots \sigma_{n-2}\right)^{-1}
$$

for $i=3, \ldots, n$. Let $\phi_{i}$ be the permutation of $\{1, \ldots, n\}$ such that $\sigma_{i} c_{j}$ is in the coset $c_{\phi_{i} j} \operatorname{Stab}\left(p_{n}\right)$. Thus $\phi_{i}$ is the transposition $(n-i, n-i+1)$. Then

$$
\sigma_{i}\left(c_{j} \cdot v\right)=c_{\phi_{i} j} \cdot\left(c_{\phi_{i} j}^{-1} \sigma_{i} c_{j} v\right),
$$

for any $i=1, \ldots, n-2, j=1, \ldots, n$ and $v \in V$. 
Let $\tau=\sigma_{1} \sigma_{2} \ldots \sigma_{n-2} \sigma_{n-2} \ldots \sigma_{2} \sigma_{1}$ and let $\nu_{j}=\sigma_{n-j+1} \sigma_{n-j+2} \ldots \sigma_{n-2}$. Then:

$$
c_{\phi_{i j} j}^{-1} \sigma_{i} c_{j}= \begin{cases}\sigma_{i} & i \neq n-1, j \neq n+1-i \\ \left(\sigma_{1} \ldots \sigma_{i-1}\right) \tau^{-1}\left(\sigma_{1} \ldots \sigma_{i-1}\right)^{-1} \sigma_{i}^{-1} & i \neq n-1, j=n+1-i \\ I d & i=n-1, j=1 \\ \sigma_{n-2} \tau^{-1} & i=n-1, j=2 \\ \nu_{j} \sigma_{n-2} \nu_{j}^{-1} & i=n-1, j>2\end{cases}
$$

One can now deduce the matrices $\mathcal{K}_{n}\left(\sigma_{i}\right)$.

\section{Remarks}

Equipped with the knowledge that the mapping class group of an arbitrarily punctured sphere is linear, Theorem 1 from $[\mathrm{BH}]$ allows us to deduce that several subgroups of mapping class groups are linear.

Let $S$ be a closed 2-manifold together with a group $G$ of covering transformations acting on it. The covering transformations are allowed to have a finite number of branch points. Let $n$ be the number of branch points of the covering space $S \rightarrow S / G$ and let $\operatorname{Diff}^{G} S$ be the group of fiber-preserving diffeomorphisms of that covering space. An easy covering space argument shows that there is an exact sequence of groups

$$
G \rightarrow \pi_{0} \operatorname{Diff}^{G} S \rightarrow \pi_{0} \operatorname{Diff}(S / G, n) .
$$

Suppose there is a faithful representation of $\pi_{0} \operatorname{Diff}(S / G, n)$. Then the above exact sequence gives a representation of $\pi_{0} \operatorname{Diff}^{G} S$ whose kernel is the image of $G$. If $G$ acts faithfully on $H_{1}(S)$ then we can obtain a faithful representation of $\pi_{0} \operatorname{Diff}^{G} S$ by taking a direct sum with the symplectic representation.

Suppose $G$ is solvable and fixes each branch point, and $S$ is not a sphere or a torus. Then [BH, Theorem 1] states that the map $\operatorname{Diff}^{G} S \rightarrow$ Diff $S$ induces an injection $\pi_{0} \operatorname{Diff}^{G} S \rightarrow \pi_{0}$ Diff $S$. We claim that $\pi_{0} \operatorname{Diff}^{G} S$ is the normalizer of $G$ in $\pi_{0}$ Diff $S$. The proof of this claim uses the fact that any element of $\pi_{0}$ Diff $S$ which normalizes the image of $G$ in $\pi_{0}$ Diff $S$ can be lifted to an element of Diff $S$ which normalizes $G$. This is proved for the case $G$ is cyclic in [BH, Theorem 3]. The general case follows exactly the same proof but uses the fact that the Nielsen realization problem is now solved for all finite groups [Ker].

The above line of reasoning can be used to obtain a faithful representation of the hyperelliptic mapping class group of a closed surface $S$. This is the group of elements of $\pi_{0}$ Diff $S$ which commute with the hyperelliptic involution. In this 
case the group $G$ is $\mathbf{Z}_{2}$, generated by the hyperelliptic involution. The quotient $S / G$ is a sphere with $2 g+2$ branch points. The generator of $G$ acts as $-I$ on $H_{1}(S)$.

More generally, if $S \rightarrow S^{2}$ is a branched covering space such that the group of covering transformations is solvable and fixes the branch points then the normalizer of $G$ in $\operatorname{Diff}(S)$ is linear. The argument proceeds as previously except we need to show that $G$ acts faithfully on $H_{1}(S)$. This follows from the well-known fact that the Torelli group is torsion-free. One way to see this is to realize a torsion element as an isometry of the surface with a suitable hyperbolic structure [Ker]. Such a map cannot be trivial on homology (see, for example [FK, Section V.3]).

Finally, note that if $S$ is a finite-sheeted covering space of $\Sigma_{2}$ without branch points, with solvable group of covering transformations, then by the same methods, we obtain a faithful representation of the normalizer of the group of covering transformations in $\pi_{0}$ Diff $S$.

\section{References}

[Big] Stephen J. Bigelow, Braid Groups are Linear. J. Amer. Math. Soc. 14 (2001), no.2, 471-486.

[BH] Joan S. Birman, Hugh M. Hilden, On Isotopies of Homeomorphisms of Riemann Surfaces, Ann. Math. (2) 97 (1973), 424-439.

[FK] H. M. Farkas, I. Kra, Riemann surfaces, 2nd ed., Graduate Texts in Mathematics, 71. Springer-Verlag, New York, 1992.

[Hir] Morris W. Hirsch, Differential topology, Graduate Texts in Mathematics, 33. Springer-Verlag, New York, 1976.

[Ker] Steven P. Kerckhoff, The Nielsen Realization Problem. Ann. Math. (2) 117 (1983), 235-265.

[Kor] Mustafa Korkmaz, On the linearity of certain mapping class groups, Turkish J. Math. 24 (2000), no. 4, 367-371.

[Kra1] Daan Krammer, The braid group $B_{4}$ is linear, Invent. Math. 142 (2000), no.3, 451-486.

[Kra2] Daan Krammer, Braid groups are linear, Ann. of Math. (2), to appear.

[Lan] Serge Lang, Algebra, 3rd ed., Addison-Wesley Publishing Co., Reading, Mass.London-Don Mills, Ont., 1993.

[Lic] W.B.R. Lickorish, A Finite Set of Generators for the Homeotopy Group of a 2-Manifold. Proc. Cambridge Philos. Soc. 60 (1964), 768-778. 
Department of Mathematics and Statistics, University of Melbourne

Parkville, Victoria, 3010, Australia

and

Department of Mathematics, Cornell University

Ithaca, New York 14853-4201, USA

Email: bigelow@unimelb.edu.au and rybu@math.cornell.edu

Received: 2 August $2001 \quad$ Revised: 15 November 2001

Algebraic 63 Geometric Topology, Volume 1 (2001) 